\title{
Les relations commerciales entre les petits producteurs hydrauliciens et EDF depuis un demi-siècle
}

\author{
Commercial relations between small hydropower productors and EDF \\ during half a century
}

par A. Goubet

Ingénieur général des P \& C, e.r.

For half a century, relationship between small power productors and EDF have been staying almost unchanged. When substantial modifications are to be introduced, commercial relationship evolution between the two entities during this period is beeing remembered.

\section{1 LE CADRE RÉSULTANT DES LOIS DE 1946 ET 1949 ET DU DÉCRET DE 1955}

A la veille de changements profonds, dont la nature et les détails sont totalement inconnus mais qui peuvent rapprocher les petits producteurs hydrauliciens des conditions qu'ils ont connu avant la nationalisation, il n'est pas inintéressant de rappeler les grandes lignes des changements survenus depuis 5 ans et qui ont conduit à la situation actuelle.

Le régime de la nationalisation, tel qu'il résulte des deux lois consécutives de 1946 et 1949, n'a rien changé quant aux conditions contractuelles qui existaient entre les sociétés de distribution et leurs fournisseurs ou leurs clients, EDF se trouvant seulement substituée aux droits et obligations de ces sociétés.

La nationalisation n'a apporté de modification brutale que pour les petits producteurs qui desservaient directement un consommateur final, puis progressivement, elle a modifié la situation des autres producteurs lorsque leurs contrats arrivaient à expiration. Il était évidemment impensable d'inciter chaque industriel à négocier directement avec la lourde entité que constituait EDF, et en 1955 intervenait le décret introduisant l'obligation d'achat par EDF, disposition constituant la contrepartie normale de l'interdiction de vente en dehors d'EDF.

Mais l'énoncé du principe ne suffisait pas car le poids des parties en présence restait disproportionné, quand bien même les producteurs se seraient regroupés pour négocier globalement. Seul l'Etat pouvait définir des "règles de jeu" nationales sous des formes diverses et en cherchant à poursuivre des objectifs qui ont varié dans le temps. Bien entendu ces règles étaient des compromis qui, comme tout bon compromis, suscitait des réserves (le terme est faible) des entités concernées.

Il convient de noter que l'on aboutissait nécessairement ainsi à une situation qui ne pourrait que provoquer les plus vives réserves sur le plan de la pure économie théoriquel : les prix de vente sont fixés indépendamment des prix de revient de chacun des acteurs économiques et peuvent donner naissance à des marges bénéficiaires extrêmement variables ; de plus, chimère pour une activité industrielle normale, la totalité de la production est écoulée. Cette situation n'est pas sans rappeler celle qui existe en matière de prix agricoles pour lesquels I'on peut, selon l'objectif recherché, insister sur la situation du petit exploitant de montagne ou sur celle du céréalier beauceron.

Mais la comparaison entre les deux domaines d'activité s'arrête là car les prix sont fixés selon des objectifs totalement différents dans les deux cas. En 1955, la production autonome était peut-être plus tolérée qu'autorisée et ne devait pas porter préjudice au consommateur final. Le prix d'achat devait donc être égal du prix de vente diminué de la marge de commercialisation supportée par le distributeur. Le principe paraît simple, clair et de bon sens... il était en fait difficile à mettre en œuvre à une époque où il existait à peu près autant de tarifs de vente en basse tension que d'anciennes sociétés (le tarif universel s'est mis en place très progressivement autour de 1960) et où le tarif vert moyenne tension supposait, pour les faibles puissances, des frais de comptage qui représentaient une part importante de la fac-

1 Qui a tendance à se retrouver actuellement à la base des mutations en cours dans le domaine électrique. 
ture totale. S'introduisaient en outre les "cas d'école" : quel est le tarif de vente à considérer pour un moulin rééquipé en centre ville dont la production est vendue en basse tension dans un rayon de quelques centaines de mètres ? ${ }^{2}$

\section{D'ÉVOLUTION RÉCENTE (À CADRE RÉGLEMENTAIRE INCHANGÉ)}

Ce principe a néanmoins subsisté jusqu'à une époque récente, les modalités d'application ayant pu éventuellement s'adapter aux évolutions de caractéristiques du parc (tendance par exemple à l'augmentation des puissances unitaires). Il a en fait duré aussi longtemps que les choix d'investissement entre hydraulique, thermique ou nucléaire ont résulté essentiellement de comparaisons économiques, voire même pendant la période où l'hydraulique était plutôt considérée comme une activité anti-écologique ("faites donc une centrale nucléaire de plus et laissez nos rivières tranquilles").

Plus récemment deux éléments nouveaux sont apparus, totalement indépendants mais dont les conséquences vont dans le même sens.

Le premier est la réduction des prix de l'électricité pour le consommateur qui constitue le grand objectif des mesures de "dérégulation" mises en place actuellement dans le monde. La France ne peut évidemment rester à l'écart d'une telle tendance même si elle envisage de l'obtenir par des dispositions spécifiques et EDF estime pouvoir réaliser une baisse des prix de vente de $25 \%$ en 5 ans. Un mouvement parallèle des prix d'achat aux producteurs hydrauliciens se traduirait pour nombre d'entre eux par des "coûts échoués" dont la prise en compte pose de sérieuses difficultés dans nombre de pays. La dernière décision gouvernementale qui est en train de se traduire par la mise en place de nouveaux contrats prévoit donc la stabilisation des prix de vente au niveau atteint en 1996, quels que soient l'âge et le degré d'amortissement des installations non nationalisées.

En outre il se dessine à travers le monde un mouvement tendant à privilégier la petite énergie hydraulique qui, sans perturber de façon excessive les conditions d'écoulement hydrographique, permet de réduire, dans une très modeste mesure il est vrai, les émissions polluantes des combustibles fossiles $^{3}$. Dorénavant, pour favoriser l'installation de nouveaux moyens ou l'amélioration des installations existantes, un supplément de prix de l'ordre de 5\% est accordé pendant 25 ans aux prix "niveau 1996". Par ailleurs cet intérêt spécifique de la petite hydraulique justifie également une évolution des prix d'achat différente de celle des prix de vente aux consommateurs.

Bien entendu on pourrait aboutir au début du prochain millénaire à des coût d'achat supérieurs d'environ $40 \%$ à ceux qui auraient résulté du maintien des errements antérieurs ${ }^{4}$. Ce résultat, voulu par les pouvoirs publics est, par sa nature, analogue aux aides accordés explicitement à tel ou tel type d'activités : production charbonnière en Allemagne. nucléaire en Angleterre, éolienne ou solaire ailleurs.

En France le mécanisme sera moins visible mais il faudra néanmoins décider, d'une manière ou d'une autre, de la façon dont cet écart de coût sera assumé : si l'on considère que la production hydraulique présente un intérêt général pour la société (diminution de l'effet de serre,...). Il serait logique de le faire supporter par tous les consommateurs quelles que soient leurs conditions d'approvisionnement mais les modalités pratiques d'application pourraient être complexes dans un contexte "dérégularisé". A l'autre extrémité de la plage des hypothèses possibles, il pourrait être supporté par les seuls consommateurs basse tension qui vont encore vraisemblablement rester longtemps les clients d'un ou de quelques grands distributeurs (les sommes en jeu

Tableau 1. Vente de courant à EDF-GDF Services entre 1970 et 1996.

\begin{tabular}{|l|c|c|c|c|c|}
\hline & \multicolumn{2}{|c|}{ production } & hydraulicité & \multicolumn{2}{c|}{ prix } \\
& $\mathrm{Gwh}^{5}$ & en $\%$ & & 27,2 & 100 \\
\hline année 1970 (référence) & 635 & 100 & 1,11 & 25,5 & 93,7 \\
\hline $1970-74$ & 703 & 111 & 0,95 & 30,2 & 111 \\
\hline $1975-79$ & 991 & 156 & 1,07 & 37,7 & 138 \\
\hline $1980-84$ & 1551 & 244 & 1,09 & 32,4 & 119 \\
\hline $1985-89$ & 2000 & 315 & 0,92 & 32,3 & 119 \\
\hline $1990-94$ & 2686 & 428 & 0,95 & 32,9 & 121 \\
\hline $1995-96$ & 3181 & 501 & 1,015 & & \\
\hline
\end{tabular}

2 Le tarif de vente servant de référence pour le tarif d'achat a toujours été le tarif 20 (ou 15) kV même pour les très petites centrales hydroélectriques. II faut toutefois se souvenir qu'au début des années 1970 EDF refusait fréquemment une fourniture d'une quinzaine de $\mathrm{kW}$ en BT, même en dehors de la zone rurale.

3 Cette approche est plus théorique que pratique en France où le fonctionnement des installations thermiques d'EDF est pratiquement réduit au strict minimum exigé par la stabilité des réseaux : une petite centrale hydraulique dans les Alpes ne modifie en rien le fonctionnement d'une centrale thermique parisienne.
4 En admettant des baisses des prix de vente du courant $20 \mathrm{kV}$ de $8 \%$ entre début 96 et fin 97 puis $6 \%$ l'année suivante et $4.5 \%$ les trois années ultérieures. II convient de remarquer que cette valeur de $40 \%$ correspond à une baisse totale de $28.5 \%(1 / 0,715=1,4)$.

5 Moyenne annuelle.

6 Il s'agit de valeurs comptables correspondant au total des factures payées par EDF divisé par le total des kilowattheures livrés. Il peut y avoir d'autres moyens de calculer des valeurs moyennes (production "en ruban"...). En francs constants 1996/prix modifiés proportionnellement au produit intérieur brut marchant (PIBM). 
pourraient correspondre à $0.5 \%$ des factures de ces derniers et resteraient sensiblement inférieures aux conséquences résultant par exemple de l'application des tarifs continentaux à la Corse et aux DOM).

\section{LES CONSÉQUENCES OBSERVÉES DEPUIS 25 ANS}

Pour conclure, et pour mettre en évidence les conséquences pratiques des mesures tarifaires du dernier demisiècle, on peut citer les valeurs des ventes de la période 1970-1996 (ensemble des producteurs hydrauliciens livrant la totalité de leur production à EDF/GDF Services). Je donne des valeurs sur 5 ans car les variations d'hydraulicité d'une année à l'autre introduisent des modifications importantes et non significatives.
La production en 26 ans a augmenté de $11 \%$ par an en moyenne, ce qui montre qu'entre les prix de vente pratiqués et les prix de revient moyens, il existe une marge que des industriels avertis peuvent exploiter avec opportunité (il est toutefois vraisemblable qu'une petite partie de cette hausse résulte de la cessation de l'activité principale de petits industriels dont la centrale hydroélectrique s'est ainsi trouvée transférée de catégorie).

Mais que personne ne cherche à me faire dire si cette marge est, ou non, excessive ! Il est certain que les tarifs pratiqués à une certaine époque ont incité de gros investisseurs à réaliser d'assez nombreuses centrales d'une puissance de quelques milliers de $\mathrm{kW}$ (le cas de la C.G.E. par exemple est bien connu). Il est possible que simultanément, et pour des aménagements de très petite puissance, ils permettent à peine un amortissement normal. 